\title{
Gender and HIV Testing Service Uptake: Trend in Northern Nigeria Onu Kema Anthony ${ }^{1 *}$, Towolawi Adetayo', Oluwasina Folajinmi' ${ }^{1}$, Onu Eugene A $^{2}$, Obioma Uchendu ${ }^{3}$, Nwakanma Ikenna ${ }^{4}$ and Ogbang Doris $^{4}$
}

${ }^{1}$ AIDS Healthcare Foundation, Nigeria

${ }^{2}$ Centre for Clinical care and Clinical Research, Nigeria

${ }^{3}$ Department of Community Medicine, University College Hospital, Ibadan, Nigeria

${ }^{4}$ African Centre for Health Leadership, Nigeria

\begin{abstract}
HIV counseling and testing remains a fundamental entry point of care in limiting transmission of HIVIAIDS. Community outreaches serves the dual function of improving access to service and outcome of care through early linkages and subsequent initiation of Anti-Retroviral Therapy (ART). Gender and socio-cultural factors affects uptake of screening services and ultimately early treatment. This study therefore assessed gender-related HIV counseling and testing uptake and linkage to care among those who were tested. A review of data from 423 community outreaches conducted between August 2012 to July 2015 by AHF Nigeria in which information on socio-demographic characteristics, HIV counseling and testing and time to accessing care was obtained. Means and proportions were used to document the results. With a monthly Mean of 2650 [male $68.8 \%$ female $38 \%$ ] clients counselled, tested and received results, $2.6 \%$ (SD 0.7) were positive; male $33.5 \%$ (SD 10) female $60.8 \%$ (SD 9). An average of $64 \%$ of positive clients were linked to care; male $40 \%$ (SD 10) female $60 \%$ (SD 10). Male predominance in testing uptake with more female positive results and early linkage to care forms a persisting trend. HIV testing activities aimed at increasing female participation by addressing social and cultural barriers limiting their participation through advocacy and community dialogue approaches would increase case detection and early linkages to care and help reduce gaps in prevention and treatment in HIV.
\end{abstract}

Keywords: HIV testing services; Gender; HIV care; Early linkage to care; Northern Nigeria

\section{Introduction}

A fundamental starting point in stopping and reversing the spread of HIV/AIDS especially in sub-Saharan Africa is HIV Testing Services (HTS).HTS includes a range of services that should be provided with HIV testing. Services range from provider-initiated testing and counselling, stand-alone voluntary counselling and testing (VCT), to home, mobile and outreach testing in the community, "moonlight" testing during evening hours in high-risk settings, in schools, workplaces, religious facilities, and transport hubs. Increasing the coverage of HTS through community outreach testing when provided within WHO's essential 5Cs: Consent, Confidentiality, Counselling, Correct test results and Connection has the benefit of early Positive client linkage to care [1]. This further improves the overall treatment outcome and reduces the risk of possible transmission of infection, while negative clients are offered other prevention models to adopt, aiding them to remain negative with an added advantage of increased HIV risk perception index which is associated with improved motivation for risk reduction $[2,3]$.

Globally, many people living with HIV are not diagnosed, and so many are not linked to treatment and care [4]. In resource-limited settings, primarily sub-Saharan Africa, it is estimated that as many as $40 \%$ of people who are diagnosed through HIV testing services are not linked to care [5-7]. Barriers that hinder or delay linkage to HIV treatment and care persist, including transportation costs and distance to the facility, stigma, fear of disclosure, staff shortages and long waiting times [8], as well as policy and legal barriers that may hinder access particularly for adolescents and key populations. Ultimately, many people with HIV are diagnosed late and start ART late, with CD4 counts below 200 cells $/ \mu$ l. Such late initiation has not decreased significantly over the past decade [9]. A combination of interventions is needed to improve linkages to prevention, treatment and care. In countries with high HIV prevalence, HIV testing rates for men are generally lower than for women. Global reporting suggests that this is because in these settings HTS is conducted mainly in reproductive health services, including antenatal care (ANC), where the routine offer of HIV testing is generally the norm. Additional approaches are needed to increase uptake of HTS among men, including the provision of HTS in settings that are more appropriate and acceptable to men, and to devise ways to encourage testing of male partners in high prevalence settings and of couples and male partners of women with HIV in all settings [10].

Nigeria is highly burdened by the epidemic of HIV/AIDS. With an estimated 3 million people living with the disease, the consequence of this position is that Nigeria risks the opportunity to achieve the goal of its development plan including the Sustainable Development Goals and the vision 20/20/20 [11]. The National HIV and AIDS and Reproductive Health Survey of 2012 found very low uptake of HIV testing in Nigeria just $23 \%$ of males and $29 \%$ of females had ever been tested for HIV. Less than $70 \%$ of these people had received their results [12].

Since then, the number of HIV Testing and Counseling (HTC) sites has increased significantly from 2,624 in 2012, to 8,308 in 2015 [13]. As a result, about $180 \%$ rise in the number of people tested was seen between these years.

According to the fact sheet of the United Nations Special Session

*Corresponding author: Onu Kema Anthony, AIDS Healthcare Foundation, Nigeria, Tel: +234 8112216147; E-mail: kemaonu@yahoo.com

Received November 14, 2016; Accepted November 24, 2016; Published November 30, 2016

Citation: Anthony OK, Adetayo T, Folajinmi O, Onu Eugene A, Uchendu O, et al (2016) Gender and HIV Testing Service Uptake: Trend in Northern Nigeria. J AIDS Clin Res 7: 638. doi: 10.4172/2155-6113.1000638

Copyright: (C) 2016 Anthony OK, et al. This is an open-access article distributed under the terms of the Creative Commons Attribution License, which permits unrestricted use, distribution, and reproduction in any medium, provided the original author and source are credited. 
on Gender and HIV, gender roles play into the course of HIV/AIDS epidemic and drive the pattern of vulnerability among men, women, boys and girls, as well as the kinds of responses observed in different communities and societies. In sub-Saharan Africa, the burden of HIV is disproportionately tilted towards women, and this has been attributed to societal and biological vulnerabilities [14]. Evidence from study on Nigeria's national HIV/AIDS response conducted in 2013 based on the secondary analysis of National AIDS and Reproductive Health Survey 2007 and 2012 suggested feminization of the HIV/AIDS epidemic, showing prevalence of infection was higher for females than male across all age groups except for the 35-39 years and the 40-44 years age groups $[15,16]$.

This study provides evidence on the pattern of HTC uptake, HIV positive cases and enrolment into care by gender through community HIV testing outreaches conducted in North-central Nigeria.

\section{Methods}

\section{Study design and procedures}

The data used in this study was from community and facility based HTC program of AIDS Healthcare Foundation (AHF) conducted in Abuja, Nasarawa, Benue and Kogi States, North-central Nigeria. The HTC program implemented five different community-based HTC approaches namely; home based testing and counselling, evening hours testing, weekend testing, church services testing and testing during outreach services where other services such as health education were offered. A joint team of nurses and trained volunteers conducted community based HTC using similar HIV testing algorithm as used in health facility HTC. Home based testing and counselling was done through door-to-door home visits by the team twice in a month in the target locations. Outreach services were conducted five days in a month and preceded by advocacy visits to community gatekeepers two days before the outreach events. The Community outreach and HIV testing services were organized by identifying strategic locations in the communities where testing posts were set up in very confidential locations. Volunteer canvassers went round the communities with public address system, entertainment to mobilize people for testing, while testing services were ongoing at the tents. Church services testing were conducted on Sundays within church compounds with the consent of the church leaders, specifically targeting the usually large congregations at the end of the church service.

Clients' linkage into care and treatment was defined as then clients presented at the project facilities with a referral form and were documented in pre-ART register after CD4 count was done. Clients who were tracked and reported to be attending care at other health facilities were also considered linked into care after confirmation with the health facility.

\section{Study setting and participants}

The study took place within the Northern-central part of Nigeria, including Abuja, Benue, Nasarawa and Kogi States, both in the rural and urban areas. The area is characterized by inadequate access to formal health, safe water, sanitation and other infrastructure; poor structural quality of housing, insecure residential status and poor health indicators. Lack of proper health services and facilities is a major problem in the slum caused by inadequate support by the government and other stakeholders. The collective effects of inadequate health services, poverty, and difficult socio-environmental conditions increase slum dwellers' vulnerability to poor health outcomes. The study participants were children and adults of all ages who underwent testing through any of the two modalities during a three-year period (20122015).

\section{Data collection, processing and analysis}

The data used in this study was collected using National Client intake forms for testing which captured outreach activities in the target communities and was done at the point of care by the various trained service providers. The two testing approaches used were provider initiated HIV testing at the health facilities and client initiated HIV testing provided at the community based testing outreaches.

Monitoring data collected using the Ministry of Health data collection registers for the period August 2012 to July 2015 were used in this study. Data collected included demographic information, HIV testing approaches, first testing or repeat testing clients, their attendance as individuals or couples, HIV status (positive, negative), linkage into care and treatment and HTC uptake in the two testing modalities. The data collected using the registers were summarized in monthly reporting tools after quality assessment before being entered into MS excel where analysis of the data were conducted.

\section{Results}

A total of 459,223 clients were tested for HIV during the period under study. Majority were tested through community based testing approaches $92.2 \%(n=423,551)$ compared to those tested through facility-based testing approach $7.8 \%(n=35,672)$.

Most of the clients tested $89.5 \%(n=410,794)$ aged above 14 years, of which majority were male (>14 years) $54.9 \%(n=252,117)$ while female clients (>14 years) comprised $34.5 \%(n=158,677)$ of the total.

In Table 1 , it is shown that in community testing approach, more clients were reached $(92.2 \%, n=423,551)$. About one-third of all the clients tested for HIV were repeat testers $(n=148,752)$ while first time testers constitute $67.6 \%(n=310,471)$. Among those that came for testing in the different sexes, $29.3 \%$ of the female clients cane for repeat testing, and that of the male were $33.8 \%$ (Figure 1 ). HIV positivity rate among clients tested at health facilities was $5.4 \%(n=35,672)$ whereas HIV positivity at community based testing was $1.9 \%(n=423,551)$. The clients confirmed to be positive $(n=11940)$ were referred for treatment and care based on proximity and patient's choice and $64 \%(n=7,642)$ of them were confirmed to be linked into care.

All clients who tested positive during health facility-based testing

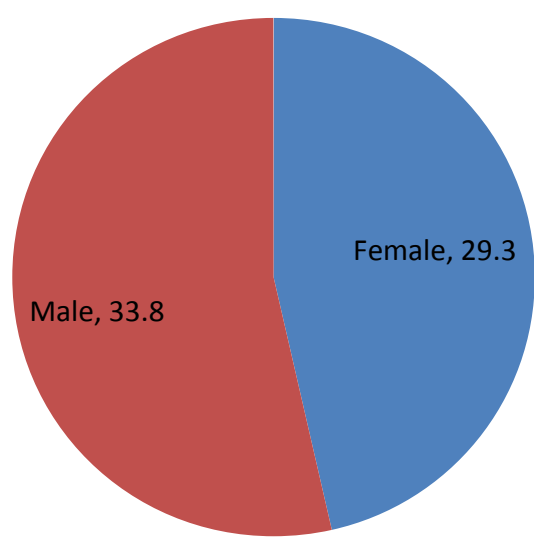

Figure 1: Percentage of participants in the different sexes who came for repeat testing in the two HTC testing models. 


\begin{tabular}{|l|c|c|c|}
\hline & Male N (\%) & Female N (\%) & Total N (\%) \\
\hline Facility testing & $15,943(44.7)$ & $19,729(55.3)$ & $35,672(7.8)$ \\
\hline Community based testing & $300,114(70.9)$ & $123,437(29.1)$ & $423,551(92.2)$ \\
\hline Positive cases linked care & $3,057(40.0)$ & $4,585(60.0)$ & $7,642(64.0)$ \\
\hline
\end{tabular}

Table 1: Pattern of HTC uptake in the different service delivery models, and linkage into care according gender.

\begin{tabular}{|l|c|c|c|}
\hline & $\begin{array}{c}\leq \mathbf{1 4} \text { Years old } \\
\mathbf{N}(\%)\end{array}$ & $\begin{array}{c}\mathbf{1 4} \text { year old } \\
\mathbf{N}(\%)\end{array}$ & Total N (\%) \\
\hline Male tested & $18,369(4.0)$ & $252,573(55.0)$ & $315,945(68.8)$ \\
\hline Female tested & $27,553(6.0)$ & $160728(35.0)$ & $143278(31.2)$ \\
\hline Positive cases (Male) & $179(1.5)$ & $2663(22.3)$ & $2,842(23.8)$ \\
\hline Positive cases (Female) & $621(5.2)$ & $8,477(71.0)$ & $9,098(76.2)$ \\
\hline
\end{tabular}

Table 2: Pattern of HIV testing uptake and testing outcome according to age groups and gender.

were successfully linked to care either at the project sites or sites of client's choice while about $64 \%$ of positive clients from outreach testing were linked to care; male $40 \%$ female $60 \%$. At health facility testing $(n=287,191)$, clients aged above 14 years had a positivity of $5.2 \%$ compared to the young people aged below or equal to 14 years whose HIV positivity was about $2.1 \%$. The HIV positivity rate among all clients $(n=459,233)$ who were tested for HIV in the program was $2.6 \%$.

Among those that accessed HTC in the facilities $(n=35,672)$, women made up of $55.3 \%$ of the clients, while the male dominated (70.9\%) among those that came for testing in the community based testing $(\mathrm{n}=423,551)$, as reported in Table 1 . A pattern observed was that though more males were tested in the overall, $76.2 \%$ of the positive cases were recorded among the female population (Table 2), while uptake of treatment and care (linkage to care) was higher among the female (Table 1).

\section{Discussion}

An analysis of the impact of gender on HIV/AIDS demonstrates the importance of mainstreaming gender into HIV programming and finding ways to strengthen women's capacity by implementing policies and programs that increase their access to education and information. Women's empowerment is vital to reversing the epidemic [17].

Evidence has shown that females bear the larger burden of HIV in Sub Saharan Africa [18] and this pattern has also been confirmed in Nigeria [19]. The situation has largely been attributed to factors like gender roles [20], stigma, fear of discrimination, costs, cultural beliefs [21], etc. Early presentation of HIV positive individuals with early commencement of ARVs has been shown to achieve better treatment outcomes. It is exigent to design strategies that will address these challenges.

This study, which was based on HTS conducted in North-Central Nigeria in facilities and through testing campaigns conducted in monthly outreaches show gender differences in uptake of these services. Data in this study suggests lower uptake of HIV testing services at the community based testing by females, but higher uptake in the facility provider initiated HIV testing. This further raises questions on the limiting factors that prevent women in northcentral Nigeria from accessing HIV services even when the barrier of proximity has been removed. A suggestion of poor health seeking behaviour would have been made, but the evidence from this study seems to prove otherwise as more HIV positive women accepted the enrolment into care compared to their male counterparts. Al-Mujtaba et al. in their study on evaluating religious influences on the utilization of maternal health services among Muslim and Christian women in North-Central Nigeria had observed similar scenario and linked it to faith-based influences on healthcare utilization among Muslim Nigerian women in which faithrelated factors were considered as barriers to Muslim women's use of maternal health services [22].

Socio-cultural factors such as polygamy, poverty, illiteracy early marriages among females, female genital mutilating practices, etc. Prevalent in these areas influence the trend in gender uptake and linkage to care in clients offered these services through community outreaches. These factors greatly affect the "sensitivity" of HIV testing services and increase the ratio of clients tested, to positive clients linked to care. Strategies that will alter this trend will give a great success story in the Nigeria HIV response. Such strategies should include; scale-up of HTS through testing campaigns and monthly outreaches with emphasis on intensified advocacy prior to these testing activities. Advocacy should focus on community sensitization and Community dialogues aimed at identifying and addressing the barriers limiting female participation in Community based testing thus increasing their participation in uptake of HTS. This will increase the "sensitivity" of this testing services to identify more positives and ultimately ensuring early client linkage to care.

Another source of worry is the lower uptake of ART by the HIV positive males in the study. This has a serious public health implication as polygamy is a common practice in the study region. The leeway societally granted to the male population, particularly in Northern Nigeria, makes it difficult for the women to exercise their sexual rights and demand for measures that will safeguard their reproductive health. This may explain the higher HIV burden in the female population as a single HIV positive male with wrong attitude to HIV prevention, can infect more women. Data from this study shows that more men came for repeat HIV test. This could be a pointer that some positive male continue to live in denial of their infection, and such men may want to do their test again hoping to have a different result.

A major lesson from this study is that socio-cultural norms that drive the HIV epidemics are common in North-Central Nigeria, and will continue to jeopardise the efforts put in arresting new HIV infections in Nigeria.

\section{References}

1. WHO (2012) Service delivery approaches for HIV testing and counselling: A strategic framework. Geneva.

2. Wanyenze RK, Kamywa MR, Fatch R, Mayanja-Kizza H, Baveewo S, et al (2013) Abbreviated HIV counselling and testing and enhanced referral to care in Uganda: A factorial randomised controlled trial. Lancet Glob Health 1: e137-e145.

3. Metsch LR, Feaster DJ, Gooden L, Schackman BR, Matheson T, et al. (2013) Effect of risk-reduction counseling with rapid HIV testing on risk of acquiring sexually transmitted infections: The AWARE randomized clinical trial. JAMA 310: 1701-1710.

4. WHO (2013) Consolidated guidelines on the use of antiretroviral drugs for treating and preventing HIV infection: Recommendations for a public health approach. Geneva.

5. Kankasa C, Carter RJ, Briggs N, Bulterys M, Chama E, et al. (2009) Routine offering of HIV testing to hospitalized pediatric patients at university teaching hospital, Lusaka, Zambia: Acceptability and feasibility. J Acquir Immune Defic Syndr 51: 202-208

6. Kranzer K, Govindasamy D, Ford N, Johnston V, Lawn SD (2012) Quantifying and addressing losses along the continuum of care for people living with HIV infection in sub-Saharan Africa: A systematic review. J Int AIDS Soc 15: 17383

7. Rosen S, Fox MP (2011) Retention in HIV care between testing and treatment in sub-Saharan Africa: A systematic review. PLoS Med 8: e1001056. 
Citation: Anthony OK, Adetayo T, Folajinmi O, Onu Eugene A, Uchendu O, et al. (2016) Gender and HIV Testing Service Uptake: Trend in Northern Nigeria. J AIDS Clin Res 7: 638. doi: 10.4172/2155-6113.1000638

Page 4 of 4

8. Kranzer K, Govindasamy D, Ford N, Johnston V, Lawn SD (2012) Quantifying and addressing losses along the continuum of care for people living with HIV infection in sub-Saharan Africa: a systematic review. J Int AIDS Soc 15: 17383

9. Siedner MJ, Ng CK, Bassett IV, Katz IT, Bangsberg DR, et al. (2015) Trends in CD4 count at presentation to care and treatment initiation in sub-Saharan Africa, 2002-2013: A meta-analysis. Clin Infect Dis 60: 1120-1127.

10. Progress report on the global health sector response (2014) World Health Organization, Geneva.

11. UNAIDS (2014) Epidemiology fact sheet on HIV and AIDS in Nigeria.

12. Nigeria Federal Ministry of Health (2013) National HIV and AIDS and reproductive health survey 2012. NARHS Plus II.

13. National AIDS and STI Control Program, Federal Ministry of Health (2015) 2015 Annual Report.

14. United Nations Special Session on HIVIAIDS Fact Sheet (2001) Gender and HIV.

15. Magadi MA (2011) Understanding the gender disparity in HIV infection across countries in sub-Saharan Africa: Evidence from the demographic and health surveys. Sociol Health IIIn 33: 522-539.
16. Nigerian National Agency for the Control of AIDS (2014) National HIVIAIDS epidemiology and impact analysis (NHEIA) report.

17. Turner T (2003) Gender and HIVIAIDS. Intentional Journal of gynaecology and obstetrics 82: 411-418.

18. UNAIDS (2013) Global report on the global AIDS epidemic. Joint United Nations Program on HIVIAIDS (UNAIDS), Switzerland, Geneva.

19. Federal Ministry of Health, National HIVIAIDS and Reproductive Health and Serological Survey, 2012 (NARHS Plus) (2013) Federal Ministry of Health Abuja, Nigeria.

20. Iyayi RF, Igbinomwanhia O, Bardi A, Iyayi OO (2011) The control of Nigerian women over their sexuality in an era of HIVIAIDS: A study of women in Edo State in Nigeria. International NGO Journal 6: 113-121.

21. Djibuti M, Zurashvili T, Kasrashvili T, Berg CJ (2015) Factors associated with HIV counseling and testing behavior among undergraduates of universities and vocational technical training schools in Tbilisi, Georgia. BMC Public Health 15: 427.

22. Al-Mujtaba M, Cornelius LJ, Galadanci H, Erekaha S, Okundaye JN, et al. (2016) Evaluating religious influences on the utilization of maternal health services among Muslim and Christian women in north-central Nigeria. BioMed Research International 8. 